\title{
Comparison of Nutritional/Inflammatory Scores as a Preoperative Predictor of Short-Term Surgical Risks in Hepatectomy for Colorectal Metastasis
}

\author{
Ryosuke Umino ${ }^{1 \#}$; Junichi Shindoh ${ }^{1,2 \# *}$, Yuta Kobayashi ${ }^{1}$, Miho Akabane ${ }^{1}$, Kazutaka Kojima ${ }^{1}$, \\ Satoshi Okubo' ${ }^{1}$ Masaji Hashimoto ${ }^{1}$
}

'Hepatobiliary-pancreatic Surgery Division, Department of Gastroenterological Surgery, Toranomon Hospital, Tokyo, Japan

${ }^{2}$ Okinaka Memorial Institute for Medical Disease, Tokyo, Japan

\section{ABSTRACT}

Background: Various nutritional/inflammatory scores reportedly correlate with surgical outcomes of abdominal surgery, while it remains inconclusive which one is the best in prediction of short-term surgical outcomes of patients with colorectal liver metastasis (CLM). Methods: Clinical records of 367 hepatectomies for 267 patients with CLM were retrospectively reviewed. Preoperative nutritional/inflammatory status was determined using 14 reported nutritional/inflammatory scores and predictive powers of these scores for short-term surgical outcomes were compared.

Results: In receiver operating characteristics curve analysis, controlling nutritional status (CONUT) score showed the highest performance in prediction of major postoperative morbidity (area under the curve [AUC], 0.650) among the tested scores and similar tendency was also confirmed in prediction of global postoperative morbidity (AUC, 0.622). Multivariate analysis confirmed that the CONUT score showed significant correlation with both global morbidity (odds ratio [OR], $1.29 ; 95 \% \mathrm{Cl}, 1.11-1.49, \mathrm{P}=0.001)$ and major morbidity (OR, $1.31 ; 95 \% \mathrm{Cl}, 1.08-1.60 ; \mathrm{P}=0.006)$. When preoperative degree of malnutrition was classified into normal, light, and moderate according to the original CONUT scoring system, short-term surgical outcomes were well stratified as follows: any morbidity, $21.8 \%$ vs. $35.1 \%$ vs. $51.9 \%$ ( $P=0.001)$; major morbidity, $6.2 \%$ vs. $11.7 \%$ vs. $29.6 \%(P=0.002)$; and postoperative hospital stay, 11 days vs. 11 days vs. 12 days $(P=0.006)$.

Conclusions: The CONUT score could be a simple and reliable predictor of short-term surgical outcomes of patients undergoing hepatectomy for CLM.

Key words: CONUT score, colorectal liver metastasis, surgery, nutrition

\section{INTRODUCTION}

Aggressive surgical approach including perioperative chemotherapy and repeat hepatectomy has increasingly been used in the field of colorectal liver metastases (CLM) because prolonged cancer-free interval achieved through a multidisciplinary treatment approach is reportedly correlated with better survival outcomes of patients with CLM (1). With advances in perioperative

\author{
*Corresponding author: \\ Junichi Shindoh M.D., Ph.D. \\ Department of Gastroenterological \\ surgery, Toranomon Hospital \\ 2-2-2 Toranomon, Minatoku \\ Tokyo 105-8470, Japan \\ Tel: +81-3-3588-1111 \\ E-mail: shindou-tky@umin.ac.jp
}

${ }^{*} \mathrm{RU}, \mathrm{JS}$ and $\mathrm{YK}$ equally contributed to this work

Received: 15.10 .2020

Accepted: 18.12.2020

Copyright () Celsius Publishing House www.sgo-iasgo.com 
management and surgical techniques, the complexity of liver surgery has increased over time $(2,3)$ and more and more poor-risk patients are becoming surgical candidates. In such context, adequate risk assessment and preemptive management are required to avoid unnecessary postoperative morbidities in the era of enhanced recovery after surgery (ERAS).

Conventionally, presence of co-morbidities, performance status, hepatic functional reserve, (4) degree of hepatectomy, $(5,6)$ cycles of preoperative chemotherapy, $(5,7)$ chemotherapy-associated liver injury, (8) number of hepatectomy, $(9,10)$ operation time, blood loss, etc. have been thought to be risk factors for postoperative morbidities after hepatectomy for CLM. In addition to these, recent studies have reported that preoperative nutritional/inflammatory status is also predictive of short-term surgical outcomes in patients undergoing liver surgery (11-13).

Although various nutritional/inflammatory scores have been reported to be associated with surgical outcomes of patients undergoing major abdominal surgery, (14-17) their predictive powers for postoperative morbidities have not yet been adequately compared so far. Therefore, this study sought to investigate the predictive powers of reported nutritional /inflammatory scores for short-term outcomes of patients undergoing hepatectomy for CLM, and tried to clarify the best assessment tool for preoperative surgical risk prediction in the era of aggressive surgical approach.

\section{METHODS}

\section{Study population}

From a prospective database maintained by the Department of Gastroenterological Surgery, Toranomon Hospital, a total of 367 hepatectomies for 267 patients with CLM performed between April 2008 and December 2018 were identified. Clinicopathological data of these 367 consecutive hepatectomies were retrospectively reviewed for analysis.

\section{Indication of surgery for colorectal liver metastases and assessment of short-term surgical outcomes}

Our indication criteria for surgical resection of CLM has been reported elsewhere (18). Briefly, preoperative hepatic functional reserve was assessed using indocyanine green (ICG) clearance test in addition to general blood examinations. Based on the volumetry of future liver remnant, curative surgical maneuver is planned to fulfill estimated ICG clearance rate of full-functioning future liver remnant of $\geq 0.05$.

Severity of postoperative morbidity was determined according to the Clavien-Dindo classification (19) and grade Illa or greater complication was classified as major morbidity.

\section{Assessment of preoperative nutritional status}

Preoperative nutritional status was determined by following 14 nutritional/hepatic functional scores/ grades using blood samples obtained within one week before surgery: Glasgow prognostic score (GPS), (20) modified GPS (mGPS),(21) Onodera's prognostic nutritional index (PNI),(22) controlling nutritional status (CONUT) score,(23) Naples prognostic score (NPS),(24) neutrophil-lymphocyte ratio (NLR),(25) plateletlymphocyte ratio $(\mathrm{PLR})$, lymphocyte-monocyte ratio (LMR), albumin-bilirubin (ALBI) score/grade, (26) albumin-indocyanine green evaluation (ALICE) score/ grade,(27) and platelet-albumin (PAL) score/grade(28) (see table S1 for details of calculation).

\section{Statistical analysis}

Statistical analysis was performed using the JMP software (version 14; SAS Institute Inc., Cary, NC) or the IBM SPSS software (Ver 26.0 SPSS Inc., Chicago, IL). Continuous values were expressed as the median (range) and were compared using the Wilcoxon's rank-sum test. Categorical variables were expressed as the number (\%) and were compared using the Fisher exact test or the chi-squared test, as appropriate. The most appropriate nutritional score for preoperative surgical risk assessment was determined among the 14 tested nutritional scores using the receiver operating characteristic (ROC) curve analysis for global and major postoperative morbidities. Then, its correlation with the short-term surgical outcomes was investigated with a multivariate analysis using logistic regression model with backward selection. To prevent overfitting, only factors that showed a statistically significant association with postoperative morbidities with $\mathrm{P}$ values $>0.1$ were included in the final model.

\section{RESULTS}

\section{Baseline characteristics}

The baseline characteristics of the study population are summarized in table 1. Median age was 63 and $67.8 \%$ of the patients were male. Of the 367 hepatectomies, 
Table S1 - Nutritional/inflammatory scoring systems

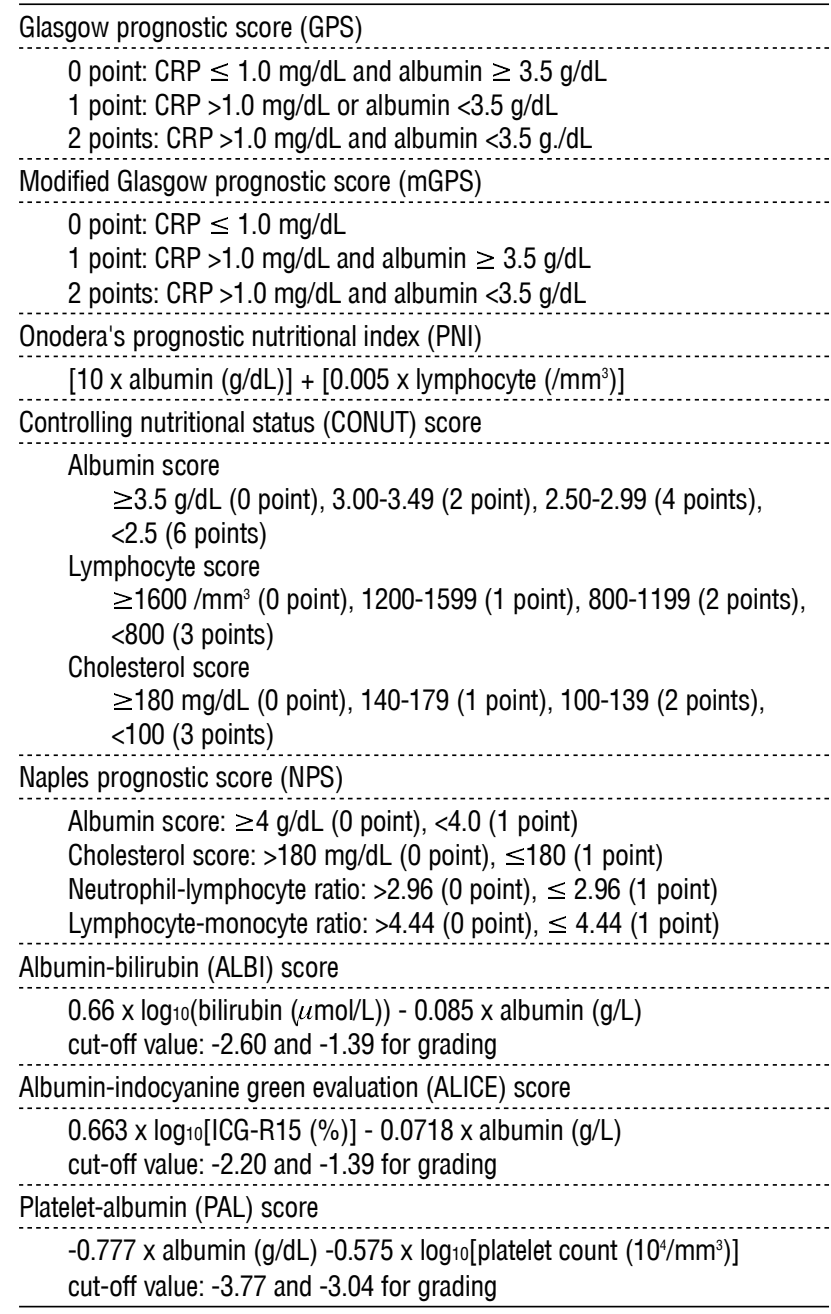

$267(72.8 \%)$ cases were initial hepatectomy and the remaining 100 (27.2\%) cases were repeat hepatectomies for recurrence or 2-stage approach for remaining tumors. Preoperative chemotherapy was used in 121 (33.0\%) cases and major hepatectomy was selected in 78 (21.2\%) cases. Median operation time was 175 minutes and estimated blood loss was $352 \mathrm{~mL}$. Incidences of any morbidity and major morbidity (i.e., Clavien-Dindo $\geq 111 a$ ) were $29.9 \%$ and $10.9 \%$, respectively.

\section{Preoperative nutritional / inflammatory scores and its performance in prediction of postoperative morbidities}

Area under the curves (AUCs) in the ROC analysis for the tested 14 scores are summarized in table 2. For prediction of any postoperative morbidity, ALICE score (AUC 0.633), PAL score (AUC 0.631), ALBI score (AUC 0.627), PNI (AUC 0.623), and CONUT score (AUC 0.622)
Table 1 - Baseline characteristics

\begin{tabular}{|c|c|}
\hline Age, $y$ & $63(31-88)$ \\
\hline Male & $249(67.8)$ \\
\hline ASA-PS, class $1 / 2 / 3$ & $160(44.4) / 182(50.6) / / 18(5.0)$ \\
\hline $\begin{array}{l}\text { Number of hepatectomy } \\
\qquad \begin{array}{l}1 \\
2 \\
3 \\
4 \\
5\end{array}\end{array}$ & $\begin{array}{r}267(72.8) \\
74(20.2) \\
17(4.6) \\
7(1.9) \\
2(0.5)\end{array}$ \\
\hline Number of tumor & $2(1-83)$ \\
\hline Maximum size of tumor (mm) & $23(5-155)$ \\
\hline Preoperative chemotherapy & $121(33.0)$ \\
\hline Albumin (g/dL) & $3.8(2.3-4.9)$ \\
\hline Total bilirubin (mg/dL) & $0.8(0.2-7.3)$ \\
\hline Total cholesterol (mg/dL) & 196 (64-399) \\
\hline C-reactive protein $(\mathrm{mg} / \mathrm{dL})$ & $0.1(0-14.3)$ \\
\hline Prothrombin activity $(\%)$ & $96(63-138)$ \\
\hline White blood cell $(/ \mathrm{mm} 3)$ & $5.2(2.8-14.9)$ \\
\hline Neutrophil (\%) & $58.8(19.0-87.5)$ \\
\hline Lymphocyte (\%) & $29.4(5.0-59.0)$ \\
\hline Monocyte $(\%)$ & $6.4(0.5-20.0)$ \\
\hline Platelet count (104/mm3) & $20.0(1.4-52.2)$ \\
\hline ICG-R15 (\%) & $9.8(0.9-81.3)$ \\
\hline Major hepatectomy & $78(21.2)$ \\
\hline Laparoscopic approach & $30(8.2)$ \\
\hline Operation time (min) & $175(47-1159)$ \\
\hline Estimated blood loss (mL) & $352(0-7359)$ \\
\hline Transfusion & $24(6.5)$ \\
\hline Any morbidity & $110(29.9)$ \\
\hline Major morbidity & $40(10.9)$ \\
\hline
\end{tabular}

showed good performance compared to the other nutritional/inflammatory scores. For major morbidity, CONUT score (AUC 0.650) showed the highest performance among the 14 scores, followed by PAL score (AUC 0.646), PNI (AUC 0.643), ALICE score (AUC 0.639), and GPS (AUC 0.633).

Given these results and clinical usability without complex calculation such as logarithm, CONUT score was selected as the best scoring system for predicting both global and major postoperative morbidities in patients undergoing hepatectomy for CLMs.

\section{Short-term outcomes according to the undernutrition grade based on the CONUT score}

When stratifying the study cohort according to the undernutrition grade of the CONUT score defined in the original report (23), 179 (49.7\%) cases were classified as 
Table 2 - Predictive powers of nutritional/inflammatory scores for postoperative morbidities

\begin{tabular}{lc}
\hline Any morbidity & AUC \\
\hline Categorical variable systems & \\
CONUT score & 0.622 \\
GPS & 0.603 \\
PAL grade & 0.587 \\
ALICE grade & 0.582 \\
NPS & 0.575 \\
ALBI grade & 0.566 \\
modified GPS & 0.501 \\
\hline Continuous variable systems & \\
ALICE score & 0.633 \\
PAL score & 0.631 \\
PNI & 0.623 \\
ALBI score & 0.618 \\
LMR & 0.605 \\
PLR & 0.547 \\
NLR & 0.541 \\
\hline Major morbidity & \\
\hdashline Categorical variable systems & \\
CONUT score & \\
GPS & 0.650 \\
PAL grade & 0.633 \\
ALICE grade & 0.622 \\
ALBI grade & 0.575 \\
NPS & 0.571 \\
modified GPS & 0.556 \\
\hdashline Continuous variable systems & 0.519 \\
PAL score & \\
PNI & 0.646 \\
ALICE score & 0.643 \\
ALBI score & 0.639 \\
LMR & 0.626 \\
PLR & 0.560 \\
NLR & 0.550 \\
\hline Abo & 0.527 \\
\hline$-\cdots-\cdots$
\end{tabular}

Abbreviations. AUC, area under the curve; GPS, Glasgow prognostic score, PNI, prognostic nutritional index; NLR, neutrophil-lymphocyte ratio; PLR, platelet-lymphocyte ratio; LMR, lymphocyte-monocyte ratio; NPS, Naples prognostic score. See Supplemental Table for details.

normal (CONUT score 0-1), 154 (42.8\%) were classified as light (CONUT score 2-4), and 27 (7.5\%) were classified as moderate (CONUT 5-8), excluding 7 cases missing total cholesterol values before surgery.

Table 3 summarized the patient characteristics and short-term surgical outcomes according to the CONUT undernutrition grade. Number and maximum diameter of tumor increased and the proportion of patients who received preoperative systemic therapy tended to be higher as the undernutrition grade got advanced. Higher proportion of patients who presented advanced CONUT score required open major hepatectomy. Incidences of any morbidity and major morbidity were well correlated with these undernutrition grade. However, there was no statistical difference regarding the other liver-related morbidities including bile leak, refractory ascites/pleural effusion, wound infection, bleeding, or postoperative hepatic insufficiency, except for abdominal abscess. The length of hospital stay also showed significant correlation with the CONUT score as follows: normal, 11 days (interquartile range [IQR], 8-15 days); light, 11 days (IQR, 9-17 days); and moderate, 12 days (IQR, $11-28$ days) ( $\mathrm{P}=0.006)$.

In multivariate analysis, CONUT score showed significant correlation with any morbidity (odds ratio [OR], 1.29; 95\% Cl, 1.11-1.49; $\mathrm{P}=0.001)$ and major morbidity (OR, 1.31; 95\% Cl, 1.08-1.60; $\mathrm{P}=0.006)$ independent from tumor factors, extent of hepatectomy, or preoperative chemotherapy. For surgical site infection, while preoperative chemotherapy and prolonged operation time were identified as independent risk factors, the CONUT score showed no significant correlation with SSI (table 4).

\section{DISCUSSION}

This study analyzed the influence of preoperative nutritional/inflammatory status on short-term surgical outcomes in patients undergoing hepatectomy for CLM. Comparison of various nutritional/inflammatory scores revealed that CONUT score, simply scored using the three variables: albumin, lymphocyte, and cholesterol, shows the best performance in prediction of postoperative morbidities. Multivariate analysis confirmed that CONUT score is associated with increased risk of both global and major morbidities, and the undernutrition grade based on the CONUT score was predictive of postoperative morbidity rates and length of hospital stay.

A glowing attention has recently been paid on nutritional status of patients undergoing treatment for cancers (29). An increased systemic immuneinflammatory response by host-tumor interaction is reportedly associated with progressive nutritional decline and may deteriorate anti-tumor immune response which results in poor oncological outcomes (30). Therefore, several studies have been conducted to establish a new evaluation method for nutritional/ inflammatory status to improve treatment outcomes for cancer patients. McMillan et al. were the first to show that the GPS, calculated by only two factors (i.e., serum levels of C-reactive protein and albumin), sensitively predicts survival outcomes of patients independent of the oncological stage or performance status (31). Josse et al. reported the importance of evaluation of inflammatory status and showed that preoperative NLR is associated with a risk of anastomotic leakage among patients undergoing resection of colorectal cancer (16). Similar studies using various scoring systems have been reported elsewhere $(14,15$, 17). Because adequate assessment of preoperative 
Table 3 - Baseline characteristics and surgical outcomes according to the CONUT undernutrition grade

\begin{tabular}{|c|c|c|c|c|}
\hline & $\begin{array}{r}\text { Normal (score 0-1) } \\
\mathrm{n}=179(49.7 \%)\end{array}$ & $\begin{array}{r}\text { Light (score 2-4) } \\
\mathrm{n}=154(42.8 \%)\end{array}$ & $\begin{array}{r}\text { Moderate (score 5-8) } \\
\mathrm{n}=27(7.5 \%)\end{array}$ & $\mathbf{P}$ \\
\hline \multicolumn{5}{|l|}{ Patent's characteristics } \\
\hline $\begin{array}{l}\text { Age, y } \\
\text { Gender, male/ female } \\
\text { ASA-PS, class } 1 / 2 / 3\end{array}$ & $\begin{array}{r}63(35-85) \\
127(71.0) / 52(29.1) \\
85(47.5) / 83(46.4) / 11(6.2)\end{array}$ & $\begin{array}{r}63(35-88) \\
105(68.2) / 49(31.8) \\
69(44.8) / 79(51.3) / 6(3.9)\end{array}$ & $\begin{array}{r}64(31-83) \\
14(51.9) / 13(48.2) \\
6(22.2) / 20(74.1) / 1(3.7)\end{array}$ & $\begin{array}{l}0.891 \\
0.138 \\
0.174\end{array}$ \\
\hline $\begin{array}{l}\text { Tumor factors } \\
\text { Primary lesion, colon/ rectum } \\
\text { Number of tumor } \\
\text { Maximum size of tumor }(\mathrm{mm}) \\
\text { Preoperative chemotherapy }\end{array}$ & $\begin{array}{r}122(68.2) / 57(31.8) \\
2(1-30) \\
20(5-125) \\
53(29.6)\end{array}$ & $\begin{array}{r}94(61.0) / 60(39.0) \\
2(1-83) \\
24(6-155) \\
54(35.1)\end{array}$ & $\begin{array}{r}14(51.9) / 13(48.2) \\
3(1-27) \\
30(8-85) \\
12(44.4)\end{array}$ & $\begin{array}{l}0.161 \\
0.116 \\
0.005 \\
0.244\end{array}$ \\
\hline $\begin{array}{l}\text { Operative parameters } \\
\text { Major hepatectomy } \\
\text { Laparoscopic approach } \\
\text { Operation time (min) } \\
\text { Estimated blood loss (mL) } \\
\text { Any morbidity (\%) } \\
\text { Bile leak } \\
\text { Ascites/Pleural effusion }\end{array}$ & $\begin{array}{r}29(16.2) \\
22(12.3) \\
169(47-584) \\
335(0-2871) \\
39(21.8) \\
12(6.7) \\
8(4.5)\end{array}$ & $\begin{array}{r}38(24.7) \\
6(3.9) \\
176(70-541) \\
373(0-3488) \\
54(35.1) \\
19(12.3) \\
16(10.4)\end{array}$ & $\begin{array}{r}11(40.7) \\
1(3.7) \\
182(60-1159) \\
520(50-7359) \\
14(51.9) \\
3(11.1) \\
4(14.8)\end{array}$ & $\begin{array}{l}0.008 \\
0.029 \\
0.370 \\
0.043 \\
0.001 \\
0.293 \\
0.109\end{array}$ \\
\hline $\begin{array}{l}\text { SSI } \\
\text { Wound infection } \\
\text { Abdominal abscess } \\
\text { Postoperative bleeding } \\
\text { PH }^{\mathrm{a}} \\
\text { Others } \\
\text { Major morbidity (\%) } \\
\text { Mortality } \\
\text { Hospital stay (days) }\end{array}$ & $\begin{array}{r}4(2.2) \\
6(3.4) \\
1(0.6) \\
2(1.1) \\
12(6.7) \\
11(6.2) \\
0(0) \\
11(8-15)\end{array}$ & $\begin{array}{r}5(3.3) \\
5(3.3) \\
2(1.3) \\
0(0) \\
13(8.4) \\
18(11.7) \\
0(0) \\
11(9-17)\end{array}$ & $\begin{array}{r}0(0) \\
6(22.2) \\
0(0) \\
0(0) \\
6(22.2) \\
8(29.6) \\
0(0) \\
12(11-28)\end{array}$ & $\begin{array}{r}0.923 \\
<0.001 \\
0.829 \\
0.541 \\
0.069 \\
0.002 \\
1 \\
0.006\end{array}$ \\
\hline
\end{tabular}

Figures represent number (percentage) or median (range).

Abbreviation: SSI, surgical site infection; PHI, postoperative hepatic insufficiency; ASA-PS class, American society of anesthesiologists physical status class.

${ }^{\text {aP }}$ ostoperative hepatic insufficiency was defined as peak total bilirubin $>7 \mathrm{mg} / \mathrm{dL}$.

Table 4 - Multivariate analysis for predictors of any morbidity, major morbidity and surgical site infection

\begin{tabular}{|c|c|c|c|c|c|c|}
\hline & $P^{a}$ & Coefficients ${ }^{\mathrm{b}}$ & SE & Wald $\chi^{2}$ & $\mathbf{O R}$ & $95 \% \mathrm{Cl}$ \\
\hline $\begin{array}{l}\text { Any morbidity } \\
\text { Preoperative chemotherapy } \\
\text { Operation time }>180 \mathrm{~min} \\
\text { CONUT score }+1 \text { point } \\
\text { Number of laparotomy }+1\end{array}$ & $\begin{array}{r}<0.001 \\
<0.001 \\
0.001 \\
0.081\end{array}$ & $\begin{array}{l}1.103 \\
1.009 \\
0.253 \\
0.293\end{array}$ & $\begin{array}{l}0.266 \\
0.260 \\
0.075 \\
0.168\end{array}$ & $\begin{array}{r}17.14 \\
15.04 \\
11.45 \\
3.05\end{array}$ & $\begin{array}{l}3.01 \\
2.74 \\
1.29 \\
1.34\end{array}$ & $\begin{array}{l}1.79-5.08 \\
1.65-4.57 \\
1.11-1.49 \\
0.97-1.86\end{array}$ \\
\hline $\begin{array}{l}\text { Major morbidity } \\
\text { Blood loss }>500 \mathrm{~mL} \\
\text { Preoperative chemotherapy } \\
\text { CONUT score }+1 \text { point } \\
\text { Number of laparotomy }+1\end{array}$ & $\begin{array}{r}<0.001 \\
<0.001 \\
0.006 \\
0.056\end{array}$ & $\begin{array}{l}1.587 \\
1.564 \\
0.273 \\
0.441\end{array}$ & $\begin{array}{l}0.433 \\
0.418 \\
0.099 \\
0.231\end{array}$ & $\begin{array}{r}13.45 \\
14.03 \\
7.61 \\
3.65\end{array}$ & $\begin{array}{l}4.89 \\
4.78 \\
1.31 \\
1.55\end{array}$ & $\begin{array}{l}2.09-11.4 \\
2.11-10.8 \\
1.08-1.60 \\
0.99-2.44\end{array}$ \\
\hline $\begin{array}{l}\text { SSI }^{\mathbf{c}} \\
\text { Preoperative chemotherapy } \\
\text { Operation time }>180 \mathrm{~min} \\
\text { Blood loss }>500 \mathrm{~mL} \\
\text { CONUT score }+1 \text { point } \\
\end{array}$ & $\begin{array}{l}0.006 \\
0.006 \\
0.095 \\
0.099 \\
\end{array}$ & $\begin{array}{r}1.533 \\
-1.446 \\
0.828 \\
0.192\end{array}$ & $\begin{array}{l}0.462 \\
0.524 \\
0.496 \\
0.117 \\
\end{array}$ & $\begin{array}{r}11.02 \\
7.60 \\
2.78 \\
2.73 \\
\end{array}$ & $\begin{array}{l}4.63 \\
0.24 \\
2.29 \\
1.21 \\
\end{array}$ & $\begin{array}{l}1.87-11.4 \\
0.08-0.66 \\
0.87-6.05 \\
0.97-1.52 \\
\end{array}$ \\
\hline
\end{tabular}

a Based on likelihood test adjusted for the other factors in the final model. ${ }^{\circ}$ Estimated coefficient for the variable and the associated standard error.

'SSI included wound infection or abdominal abscess.

Abbreviations: SSI, surgical site infection; SE, standard error; OR, odds ratio; $95 \% \mathrm{Cl}, 95 \%$ confidence interval;

ASA-PS class, American society of anesthesiologists physical status class.

Note. Multivariate logistic regression was applied with stepwise backward selection. Initially all the potential independent variables were included in the statistical model. Then factors that showed no or limited statistically significant association $(P>0.1)$ adjusted for the remaining factors in the model were deleted from the model in stepwise fashion. The 10 tested variables were as follows: age (>65 vs. $\leq 65)$, gender, ASA-PS class $(\geq 2 \mathrm{vs.}<2)$, preoperative chemotherapy $(\mathrm{y} / \mathrm{n})$, number of laparotomy, degree of hepatectomy (major vs. minor), laparoscopic approach (y/n), operation time (> vs. $\leq 180$ min), estimated blood loss (> vs. $\leq 500 \mathrm{~mL}$ ), and CONUT score.

nutritional status is helpful to know the perioperative risks of patients undergoing abdominal surgery, it could also contribute to better surgical management and improved adherence to recovery protocol in the era of ERAS (32).
With a development of multidisciplinary treatment approach, more and more patients with advanced colorectal cancer including CLM are becoming surgical candidates after intensive chemotherapy. While perioperative chemotherapy and repeated hepatectomy 
contributes to better survival outcomes for $C L M,(1)$ the complexity of liver surgery and aggressive chemotherapy still remain the main cause of morbidity and mortality $(5,33,34)$. To ensure perioperative safety, meticulous preoperative workup including assessment of hepatic functional reserve and 3D volumetry has conventionally been performed to mainly avoid the risk of postoperative hepatic insufficiency $(18,35,36)$ However, given the increasing awareness of the importance of nutritional/inflammatory status, preoperative assessment would need to be more comprehensive to adequately assess the surgical risks based on the patients' physical status.

This study aimed to compare the performance of various nutritional/inflammatory scores and to investigate the most efficient evaluation method from the standpoint of reliability and usability. Of course, nutritional scores using continuous variables generally tend to show good performance. However, the noteworthy result of the present study is that nutritional scores using simple categorical variables are not always inferior to those using continuous variables requiring complex calculations. In the field hepatology, ALBI score (26) has gradually been used as an alternate method to evaluate hepatic functional reserve. However, the major issue of this score includes its complex calculation using logarithm and arbitrary cut-off points using 25 percentile and 90 percentiles for grading, though its performance has been validated in various cohorts (37, 38). The CONUT score selected as a potentially effective assessment tool for prediction of short-term surgical outcomes was relatively simple using three variables and it showed acceptable performance in prediction of any morbidity, major morbidity, and postoperative hospital stay (table 3). Although the clinical evidence regarding the use of CONUT score in preoperative assessment remains insufficient, $(15,39)$ it would be practically important that surgical risks of patients undergoing hepatectomy for CLM could be predicted relatively easily through a simple scoring system avoiding complex calculation.

The limitations of the current study include its retrospective design and inherent potential bias in a selected population. However, the current analysis was performed using a prospectively collected database of patients who were treated similarly during the study period. Additionally, it is difficult to evaluate the influence of preoperative chemotherapy on nutritional /inflammatory status and the impact of perioperative nutritional support on surgical outcomes in the present cohort. As presented in table 3, advanced undernutrition grade was correlated with advanced tumor extension, higher proportion of preoperative therapy, and higher extent of hepatectomy. Although multivariate analysis (table 4) demonstrated that the CONUT score was independently correlated with increased incidence of morbidities after hepatectomy for CLM, care should be paid in interpretation of the present results considering the differences in baseline characteristics among the three groups of patients classified according to the CONUT score. Future analysis including prospective study looking at the impact of perioperative nutritional management would be needed to further optimize the ERAS protocol.

\section{CONCLUSION}

In conclusion, the current study confirms that short-term surgical outcomes of patients undergoing hepatectomy for CLM is correlated with preoperative nutritional status and the perioperative surgical risk may be predicted relatively easily using the CONUT score.

\section{Disclosure statement}

The authors have no conflict of interest to disclose. This study was supported by a grant from Okinaka Memorial Institute for Medical Disease.

\section{Ethical approval}

This study was conducted in accordance with the Declaration of Helsinki and the ethical guidelines for clinical study in Japan under approval of the institutional review board of Toranomon Hospital (No. 1919).

\section{REFERENCES}

1. Oba M, Hasegawa K, Matsuyama Y, Shindoh J, Mise Y, Aoki T, et al. Discrepancy between recurrence-free survival and overall survival in patients with resectable colorectal liver metastases: a potential surrogate endpoint for time to surgical failure. Ann Surg Oncol. 2014;21(6):1817-24

2. Zimmitti G, Roses RE, Andreou A, Shindoh J, Curley SA, Aloia TA, et al. Greater complexity of liver surgery is not associated with an increased incidence of liver-related complications except for bile leak: an experience with 2,628 consecutive resections. J Gastrointest Surg. 2013;17(1):57-64; discussion p -5.

3. Shindoh J, Tzeng CW, Aloia TA, Curley SA, Huang SY, Mahvash A, et al. Safety and efficacy of portal vein embolization before planned major or extended hepatectomy: an institutional experience of 358 patients. J Gastrointest Surg. 2014;18(1):45-51.

4. Makuuchi M, Kosuge T, Takayama T, Yamazaki S, Kakazu T, Miyagawa S, et al. Surgery for small liver cancers. Semin Surg Oncol. 1993:9(4):298-304.

5. Shindoh J, Tzeng CW, Aloia TA, Curley SA, Zimmitti G, Wei SH, et al. Optimal future liver remnant in patients treated with extensive preoperative chemotherapy for colorectal liver metastases. Ann Surg Oncol. 2013;20(8):2493-500 
6. Kishi Y, Abdalla EK, Chun YS, Zorzi D, Madoff DC, Wallace MJ, et al. Three hundred and one consecutive extended right hepatectomies: evaluation of outcome based on systematic liver volumetry. Ann Surg. 2009;250(4):540-8.

7. Karoui M, Penna C, Amin-Hashem M, Mitry E, Benoist S, Franc B, et al. Influence of preoperative chemotherapy on the risk of major hepatectomy for colorectal liver metastases. Ann Surg. 2006 243(1):1-7.

8. Vauthey JN, Pawlik TM, Ribero D, Wu TT, Zorzi D, Hoff PM, et al Chemotherapy regimen predicts steatohepatitis and an increase in 90-day mortality after surgery for hepatic colorectal metastases. J Clin Oncol. 2006;24(13):2065-72.

9. Kobayashi $\mathrm{Y}$, Shindoh J, Igata $\mathrm{Y}$, Okubo S, Hashimoto M. A novel scoring system for evaluating the difficulty of lysis of adhesion and surgical risk at repeat hepatectomy. J Hepatobiliary Pancreat Sci. 2020;27(4):191-9.

10. Mise Y, Hasegawa K, Shindoh J, Ishizawa T, Aoki T, Sakamoto Y, et al The Feasibility of Third or More Repeat Hepatectomy for Recurrent Hepatocellular Carcinoma. Ann Surg. 2015;262(2):347-57.

11. Sungurtekin $H$, Sungurtekin U, Balci C, Zencir M, Erdem E. The influence of nutritional status on complications after major intraabdominal surgery. J Am Coll Nutr. 2004;23(3):227-32.

12. van den Broek MA, Olde Damink SW, Dejong CH, Lang H, Malago M Jalan $\mathrm{R}$, et al. Liver failure after partial hepatic resection: definition, pathophysiology, risk factors and treatment. Liver Int. 2008;28(6): 767-80.

13. Read JA, Choy ST, Beale PJ, Clarke SJ. Evaluation of nutritional and inflammatory status of advanced colorectal cancer patients and its correlation with survival. Nutr Cancer. 2006;55(1):78-85

14. Kanda M, Fujii T, Kodera Y, Nagai S, Takeda S, Nakao A. Nutritional predictors of postoperative outcome in pancreatic cancer. Br J Surg. 2011;98(2):268-74.

15. Yoshida N, Baba Y, Shigaki H, Harada K, Iwatsuki M, Kurashige J, et al. Preoperative Nutritional Assessment by Controlling Nutritional Status (CONUT) is Useful to estimate Postoperative Morbidity After Esophagectomy for Esophageal Cancer. World J Surg. 2016;40(8): 1910-7.

16. Josse JM, Cleghorn MC, Ramji KM, Jiang H, Elnahas A, Jackson TD et al. The neutrophil-to-lymphocyte ratio predicts major perioperative complications in patients undergoing colorectal surgery. Colorectal Dis. 2016;18(7):0236-42.

17. Toiyama Y, Shimura T, Yasuda H, Fujikawa H, Okita Y, Kobayashi M, et al. Clinical Burden of C-Reactive Protein/Albumin Ratio Before Curative Surgery for Patients with Gastric Cancer. Anticancer Res. 2016;36(12):6491-8

18. Kobayashi $Y$, Kiya $Y$, Nishioka $Y$, Hashimoto $M$, Shindoh J. Indocyanine green clearance of remnant liver (ICG-Krem) predicts postoperative subclinical hepatic insufficiency after resection of colorectal liver metastasis: theoretical validation for safe expansion of Makuuchi's criteria. HPB (Oxford). 2020;22(2):258-64.

19. Clavien PA, Barkun J, de Oliveira ML, Vauthey JN, Dindo D, Schulick $\mathrm{RD}$, et al. The Clavien-Dindo classification of surgical complications five-year experience. Ann Surg. 2009;250(2):187-96.

20. Forrest LM, McMillan DC, McArdle CS, Angerson WJ, Dunlop DJ Evaluation of cumulative prognostic scores based on the systemic inflammatory response in patients with inoperable non-small-cell lung cancer. Br J Cancer. 2003;89(6):1028-30.

21. McMillan DC, Crozier JE, Canna K, Angerson WJ, McArdle CS Evaluation of an inflammation-based prognostic score (GPS) in patients undergoing resection for colon and rectal cancer. Int $\mathrm{J}$ Colorectal Dis. 2007;22(8):881-6.

22. Onodera T, Goseki N, Kosaki G. [Prognostic nutritional index in gastrointestinal surgery of malnourished cancer patients]. Nihon Geka Gakkai Zasshi. 1984;85(9):1001-5.
23. Ignacio de Ulibarri J, Gonzalez-Madrono A, de Villar NG, Gonzalez P, Gonzalez B, Mancha A, et al. CONUT: a tool for controlling nutritional status. First validation in a hospital population. Nutr Hosp. 2005;20(1):38-45

24. Galizia G, Lieto E, Auricchio A, Cardella F, Mabilia A, Podzemny V, et al. Naples Prognostic Score, Based on Nutritional and Inflammatory Status, is an Independent Predictor of Long-term Outcome in Patients Undergoing Surgery for Colorectal Cancer. Dis Colon Rectum. 2017;60(12):1273-84.

25. Zahorec R. Ratio of neutrophil to lymphocyte counts--rapid and simple parameter of systemic inflammation and stress in critically ill. Bratisl Lek Listy. 2001;102(1):5-14.

26. Johnson PJ, Berhane S, Kagebayashi C, Satomura S, Teng M, Reeves $\mathrm{HL}$, et al. Assessment of liver function in patients with hepatocellular carcinoma: a new evidence-based approach-the ALBI grade. J Clin Oncol. 2015;33(6):550-8.

27. Kokudo T, Hasegawa K, Amikura K, Uldry E, Shirata C, Yamaguchi T, et al. Assessment of Preoperative Liver Function in Patients with Hepatocellular Carcinoma - The Albumin-Indocyanine Green Evaluation (ALICE) Grade. PLoS One. 2016;11(7):e0159530.

28. Shindoh J, Kawamura Y, Kobayashi Y, Kiya Y, Sugawara T, Akuta $\mathrm{N}$, et al. Platelet-Albumin Score as a Sensitive Measure for Surgical Risk Prediction and Survival Outcomes of Patients with Hepatocellular Carcinoma. J Gastrointest Surg. 2019;23(1): 76-83.

29. Arends J, Bachmann P, Baracos V, Barthelemy N, Bertz H, Bozzetti F, et al. ESPEN guidelines on nutrition in cancer patients. Clin Nutr. 2017;36(1):11-48.

30. Baracos VE. Cancer-associated malnutrition. Eur J Clin Nutr. 2018;72(9):1255-9.

31. McMillan DC. The systemic inflammation-based Glasgow Prognostic Score: a decade of experience in patients with cancer. Cancer Treat Rev. 2013;39(5):534-40.

32. Arends J, Baracos V, Bertz H, Bozzetti F, Calder PC, Deutz NEP, et al. ESPEN expert group recommendations for action against cancerrelated malnutrition. Clin Nutr. 2017;36(5):1187-96.

33. Kishi Y, Zorzi D, Contreras CM, Maru DM, Kopetz S, Ribero D, et al. Extended preoperative chemotherapy does not improve pathologic response and increases postoperative liver insufficiency after hepatic resection for colorectal liver metastases. Ann Surg Oncol. 2010;17(11):2870-6

34. Cauchy F, Aussilhou B, Dokmak S, Fuks D, Gaujoux S, Farges O, et al. Reappraisal of the risks and benefits of major liver resection in patients with initially unresectable colorectal liver metastases. Ann Surg. 2012;256(5):746-52; discussion 52-4.

35. Mise Y, Satou S, Shindoh J, Conrad C, Aoki T, Hasegawa K, et al. Three-dimensional volumetry in 107 normal livers reveals clinically relevant inter-segment variation in size. HPB (0xford). 2014;16(5): 439-47.

36. Abdalla EK, Denys A, Chevalier P, Nemr RA, Vauthey JN. Total and segmental liver volume variations: implications for liver surgery. Surgery. 2004;135(4):404-10.

37. Hiraoka A, Kumada T, Kudo M, Hirooka M, Tsuji K, Itobayashi E, et al. Albumin-Bilirubin (ALBI) Grade as Part of the EvidenceBased Clinical Practice Guideline for HCC of the Japan Society of Hepatology: A Comparison with the Liver Damage and ChildPugh Classifications. Liver Cancer. 2017:6(3):204-15.

38. Fagenson AM, Gleeson EM, Pitt HA, Lau KN. Albumin-Bilirubin Score vs Model for End-Stage Liver Disease in Predicting PostHepatectomy Outcomes. J Am Coll Surg. 2020;230(4):637-45.

39. Tokunaga R, Sakamoto Y, Nakagawa S, Ohuchi M, Izumi D, Kosumi $\mathrm{K}$, et al. CONUT: a novel independent predictive score for colorectal cancer patients undergoing potentially curative resection. Int $\mathrm{J}$ Colorectal Dis. 2017;32(1):99-106. 